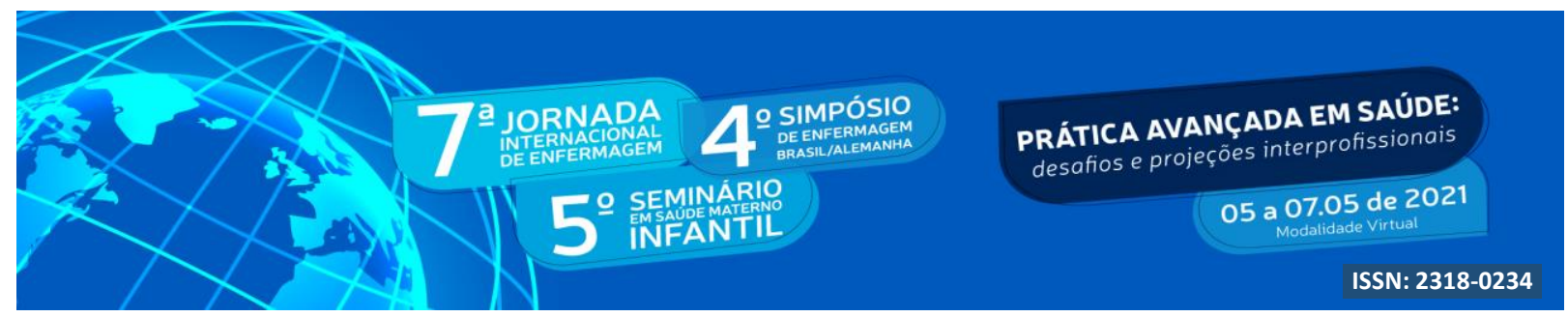

DOI: http://doi.org/10.48195/jie2021-154

\title{
HIV NA GESTAÇÃO: UM RELATO DE EXPERIÊNCIA NO SERVIÇO DE ATENDIMENTO ESPECIALIZADO
}

\author{
Nagianne Oliveira dos Santos ${ }^{1}$; Angelo Ramos Junior ${ }^{2}$; Emili Nascimento Silveira ${ }^{3}$; \\ Luiza Oliveira da Silva ${ }^{4}$; Melissa Caroline Hermann Dias ${ }^{5}$; Fabiana Porto da Silva ${ }^{6}$.
}

\begin{abstract}
RESUMO
O presente estudo expõe a vivência de residentes de Infectologia e Neurologia frente a assistência realziada à gestantes diagnosticadas com o vírus do HIV no Serviço de Atendimento Especializado (SAE). Esse trabalho possui uma abordagem descritiva e qualitativa e caracteriza-se como um relato de experência a partir da inserção de residentes do primeiro ano no SAE municipal. A partir de dados epidemiológicos do município se percebeu o grande aumento de gestantes diagnosticadas pelo vírus do HIV e a partir disso a importância de realizar um acolhimento humanizado para que essa gestante venha a criar vínculo com o serviço melhorando assima sua adesão ao tratamento. Conclui-se que a capacitação de profissionais da saúde para atender essa população é essencial e a importância de vinculação do profissional com a gestante, otimizando os atendimentos prestados e favorecendo para que os objetivos sejam alcançados.
\end{abstract}

Palavras-chave: HIV; Acolhimento; Gestante.

\begin{abstract}
The present study exposes the experience of residents of Infectious Diseases and Neurology in view of the assistance provided to pregnant women diagnosed with the HIV virus in the Specialized Care Service (SAE). This work has a descriptive and qualitative a provided to pregnant women approach and is characterized as an experience report from the insertion of first year residents in the municipal SAE. Based on epidemiological data from the municipality, there was a great increase in pregnant women diagnosed with the HIV virus and from that the importance of carrying out a humanized reception for this pregnant woman to create a bond with the service thus improving her adherence to treatment. It is concluded that the training of health professionals to serve this population is essential and the importance of linking the professional with the pregnant woman, optimizing the services provided and favoring the objectives to be achieved.
\end{abstract}

\footnotetext{
${ }^{1}$ Enfermeira. Residente Infecto e Neuro. UFN. ng.oliver@ hotmail.com

${ }^{2}$ Enfermeiro. Mestre em Enfermagem. SEG. angeloramosjunior@gmail.com

${ }^{3}$ Psicóloga. Residente Infecto e Neuro. UFN. emilipsico@ yahoo.com.br

${ }^{4}$ Fisioterapeuta. Residente Infecto e Neuro. UFN. luizaoliveira094@hotmail.com

${ }^{5}$ Psicóloga. Residente Infecto e Neuro. UFN. melissahermannpsi@ gmail.com

${ }^{6}$ Enfermeira. Tutora da Residência Infecto e Neuro. UFN. fabianaporto54@gmail.com
} 


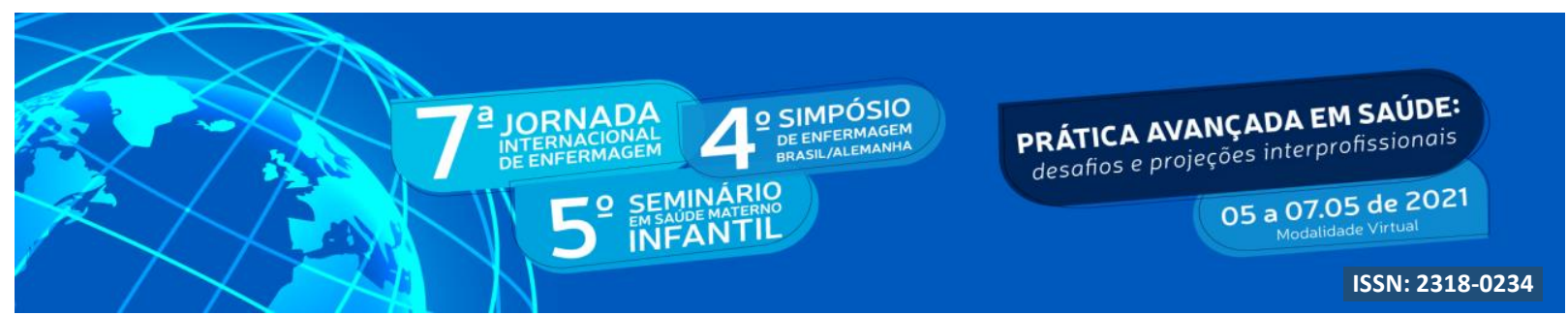

Key Words: HIV, Host, Pregnant.

\section{INTRODUÇÃO}

O vírus da imunodeficiência humana (HIV) atinge os linfócitos, principalmente as células TCD4 que são responsáveis pela defesa e proteção do nosso organismo, logo ele destrói o sistema imunológico, agravando a infecção e causado a Aids (SILVA et al, 2016).

No Brasil os primeiros casos de Aids aconteceram nos anos 80, quando ainda era considerada uma infecção pertencente a determinados grupos da população, chegando a ser chama de doença dos $5 \mathrm{H}$, pois considerava-se que as pessoas homossexuais, hemofílicos, haitianos, usuários de heroína e hookers (profissionais do sexo), seriam as únicas acometidas pelo vírus. No entanto em 1985 surgiu o primeiro caso de transmissão vertical, o que possibilitou a conclusão de que heterossexuais também poderiam ser diagnosticados com o vírus (BRITO, CASTILHO e SCZH, 2001).

Desde o surgimento o perfil epidemiológico vem sofrendo mudanças, hoje se observa que a infecção pode estar presente em todos os lugares, gêneros e idades. Para Ayla, Moreira e Francelino (2016) o aumento de casos da infecção pelo vírus do HIV, apresenta transformação epidemiológica importante, podendo se observar o aumento de casos no sexo feminino, jovens, de diferentes regiões e classes sociais.

Foi a partir do ano de 2014 que a notificação para o HIV passou a ser realizada de forma compulsória. Do ano de 2007 até junho de 2020, foram notificados no Sistema de Informação de Agravos de Notificação (SISAN) 342.459 casos de infecção pelo HIV no Brasil. Já os casos em gestantes no período do ano 2000 até junho de 2020 foram notificadas 134.328 em gestantes no Brasil, sendo 8312 só no ano de 2019. A região sul do país só fíca atrás da região sudeste em casos de HIV em gestantes, contabilizando $26,6 \%$ dos casos (BRASIL, 2020).

No ano de 2012 a Rede Cegonha foi implementada no Sistema Único de Saúde (SUS), chegando como uma estratégia do Ministério da Saúde para implementar uma rede de cuidado às mulheres, com a finalidade de estruturar e organizar a atenção à saúde materno infantil no 


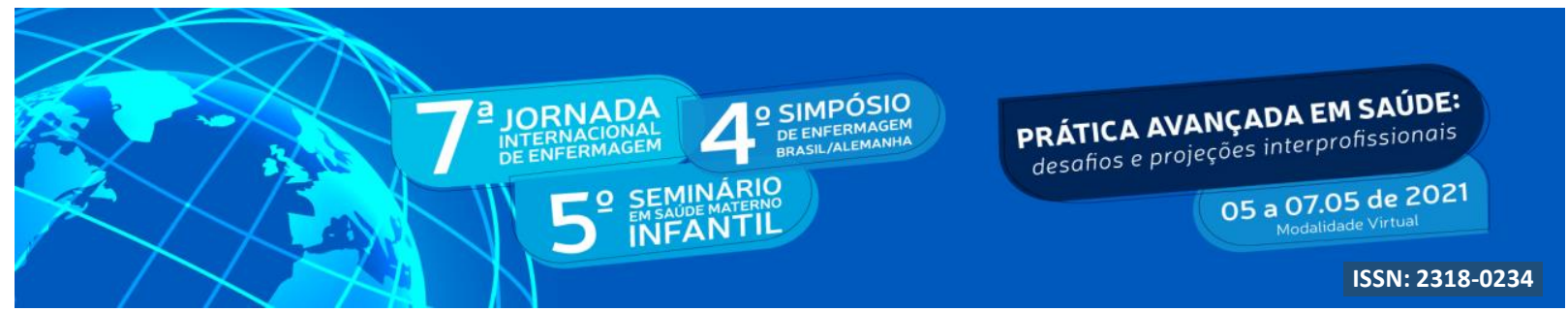

país, sendo o pré-natal um dos componentes da Rede Cegonha, que contribui para o aumento de diagnósticos de infecções sexualmente transmissíveis (IST’s) em gestantes (brasil, 2012).

Desde então fica preconizado pelo Ministério da Saúde a realização de testagem para todas as sorologias durante a gestação, com o objetivo de rastrear essas gestantes, possibilitando um tratamento imediato, oportunizando uma melhor qualidade de vida para essa mulher e ainda evitando que ocorra a transmissão vertical do HIV. Os testes já devem ser realizados na primeira consulta de pré-natal, sendo repetidos no último trimestre da gestação, ou antes caso necessário. Na falta de um pré-natal adequado, ainda existe a possibilidade de que o diagnóstico ocorra na maternidade, no momento do parto (BRASIL, 2019).

O momento do diagnóstico para uma patologia como o HIV/Aids é bastante singular para cada indivíduo, e a maneira como é informado pode contribuir para um melhor entendimento e aceitação. Sendo ainda, o acolhimento, um momento para que se estabeleça o vínculo e a humanização da assistência

\section{OBJETIVO}

O objetivo desse trabalho é relatar a experiência acerca das atividades desenvolvidas por residentes do Programa de Residência em Atenção Clínica Especializa com Ênfase em Infectologia e Neurologia, a partir da inserção no campo do Serviço de Atendimento Especializado (SAE) do município de Santa Maria -RS, durante o ano de 2020, descrevendo o processo de acolhimento e orientações por parte da equipe multiprofissional à pessoa que vive com HIV.

\section{METODOLOGIA}

Trata-se de um estudo descritivo, do tipo relato de experiência, desenvolvido por equipe multiprofissional de residentes, no SAE municipal, durante o primeiro ano de residência.

O relato de experiência é um tipo de estudo que irá promover uma reflexão sobre todas as atividades realizadas ou ações que demostram uma determinada vivência no âmbito 


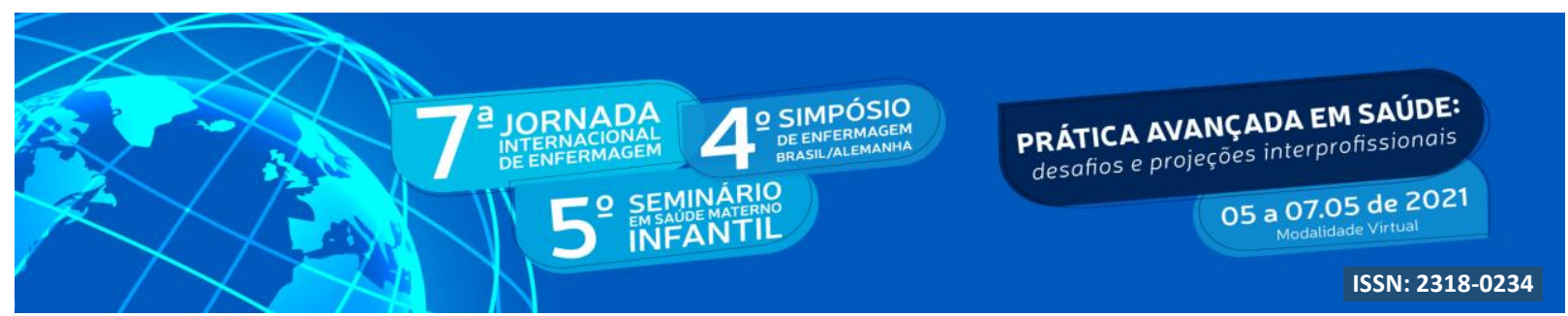

profissional, utilizando uma pesquisa qualitativa (AIRES e GUIMARÃES, 2017).

O campo de prática é um SAE, que realiza os atendimentos às pessoas que vivem com HIV/Aids, hepatite virais e outras IST's, através da realização de testes rápidos, aconselhamento pré e pós testes, acolhimento, tratamento e acompanhamento dessas infecções, sendo que serão atendidas as pessoas diagnosticadas no próprio serviço, assim como as diagnosticadas em Unidades Básicas de Saúde (UBS), Estratégias de Saúde da Família (ESF) do município, ou ainda transferências de outros municípios. Esse serviço conta com uma equipe multiprofissional, sendo enfermeiro, técnico em enfermagem, psicólogo, assistente social, nutricionista, farmacêutica, assistentes de laboratório, médico dermatologista e médicos infectologistas.

Durante o acolhimento, o profissional de saúde recebe essa gestante com o objetivo de lhe ouvir, principalmente os seus medos e dúvidas que estão relacionados ao diagnóstico e até mesmo sobre a própria gestação. A gestante é orientada sobre a infecção, sobre como o vírus irá agir em seu organismo, quais são as formas de transmissão, como é realizado o tratamento, a importância de ter uma boa adesão, explicando que aderir ao tratamento não está relacionado apenas ao uso correto da medicação, mas também a presença nas consultas médicas assim como a realização de exames de rotina.

Ainda nesse primeiro encontro é realizada a notificação, no caso de gestantes são realizadas duas notificações, uma que seria a principal da usuária e outra para a gestação, assim caso a mesma mulher venha a ter mais gestações, em cada uma delas será realizada uma nova notificação. Após se faz todos os encaminhamentos para realização de exames e agendamento da consulta médica com infectologista. É nesse momento também que pode ser ofertado os demais serviços disponíveis, assim como assistência social, os atendimentos psicológicos ou nutricionais, conforme a demanda apresentada pela gestante.

\section{RESULTADOS E DISCUSSÃO}

Durante a prática vivenciada por residentes no SAE municipal, destacou-se a importância do acolhimento às gestantes com o diagnóstico de HIV. O Boletim Epidemiológico de 2019, trouxe que o município de Santa Maria apresentou no SINAN 46 


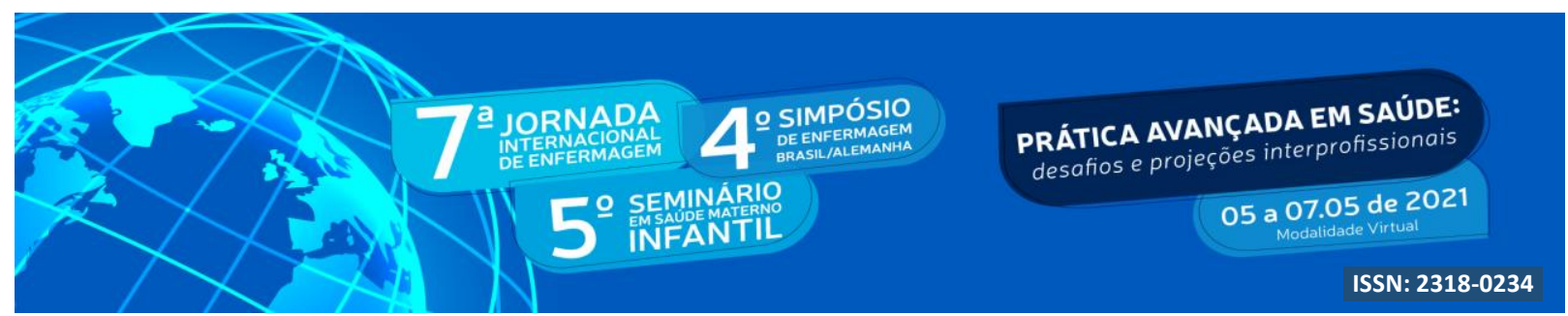

casos de notificações em gestantes (SECRETARIA MUNICIPAL DE SAÚDE, 2019).

O acolhimento de uma gestante é tão importante quanto de qualquer outro usuário, porém para Costa et al, 2019, a partir do momento que se descobre um diagnóstico na gestação, a mulher passa a ter que a lidar com a aceitação da sua própria patologia, que carrega um grande estigma e preconceito, muitas vezes por parte da própria gestante, mas também existe o medo de estra transmitindo a infecção para o filho.

No município de Santa Maria, é pactuado que a gestantes devem manter o vínculo com o serviço especializado, onde serão realizadas as consultas com infectologista, mas que também ocorra o vínculo com a UBS ou ESF de referência para dar continuidade no pré-natal e para suas demais demandas.

O cuidado de uma pessoa diagnosticada com HIV já tem início desde o teste positivo para a patologia. Para o Programa Conjunto das Nações Unidas sobre HIV/Aids - UNAIDS (2015), o tratamento vai muito além do uso da medicação, inclui todos os serviços prestados pela atenção básica e especializada, sendo fundamental o atendimento realizado por uma equipe multiprofissional.

Um ponto que merece destaque no atendimento às gestantes com HIV, é o fluxo municipal dos atendimentos, fator que muitas vezes pode contribuir para a boa ou má adesão da usuária. No município a Secretaria Municipal de Saúde (SMS) determina que a porta de entrada da gestante seja a UBS ou ESF, onde se tem início ao pré-natal, logo onde serão realizados os testes rápidos para a detecção de IST's, sendo que em casos de sorologia positiva a gestante será automaticamente encaminhada ao serviço de atendimento especializado, nesse caso a Casa Treze de Maio. Nesse serviço então que ocorre o acolhimento, seguido de consultas para o tratamento da infecção por HIV.

No serviço especializado o primeiro atendimento à gestante costuma acontecer por uma enfermeira, psicóloga ou assistente social. Talvez seja esse o primeiro momento que a gestante vai expor suas dúvidas e medos, sempre é ofertado um espaço onde a gestante possa externar todos os seus sentimentos, porem cada atendimento é único, com usas particularidades, e o profissional de saúde precisa escolher meios para que o acolhimento ocorra da forma mais satisfatória possível. Para Alves et al, 2020, esse é momento de compreender como a gestante está recebendo as informações e também de ter um olhar mais 


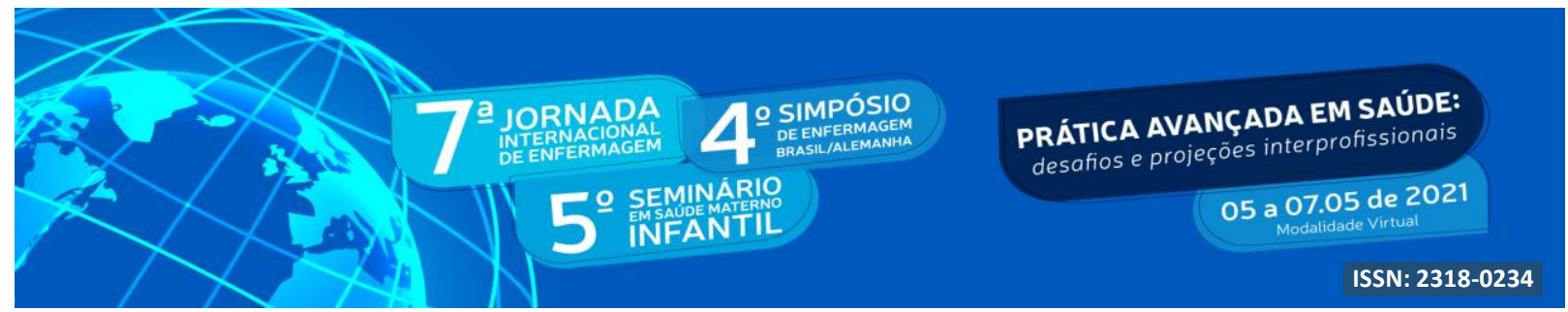

ampliado da situação, buscando entender qual o impacto esse diagnóstico está trazendo para a vida daquela gestante.

Com a vivência o que se pode observar, é que existem fatores que podem comprometer o tratamento, e o fluxo também pode acabar sendo um desses fatores, pois fica estabelecido pela SMS que a gestante além de vínculo com UBS ou ESF, e com o SAE, precisa ainda ser referenciada ao Hospital Universitário de Santa Maria (HUSM), onde será recebida pelo Ambulatório de Gestação de Alto Risco (AGAR). O que se vê na prática é que por diversas vezes a gestante se perde em meio a tantas consultas em locais diferentes, e por esse motivo o fluxo vem sendo revisto por parte da gestão municipal.

Diversos fatores podem contribuir para a má adesão ao tratamento nas gestantes, entre eles a baixa escolaridade, a falta de uma rede de apoio, o abandono por parte dos companheiros, algumas crenças, fatores culturais, falta de vínculo com o serviço de saúde e com os profissionais que ali estão e até mesmo a questão financeira (BARROS et al, 2011).

Todos esses fatores foram vistos na prática, com grande destaque em conseguir trazer para o serviço as gestantes com algum tipo de envolvimento com as drogas ou que vivem em extrema vulnerabilidade, pois o que se constata é a grande dificuldade de entender o seu próprio diagnóstico, de entender a importância de estra realizando um tratamento adequado e a dificuldade financeira que impossibilita muitas vezes a gestante de estar se deslocando por diversos locais de atendimento. Para Santos (2016) o acolhimento deve ser conduzido a parir de uma escuta qualificada e atenta as demandas do usuário, pois não basta ter todo o saber sobre a parte clínica da doença, saber os melhores tratamentos e deixarmos as angústias e inquietações do usuário em segundo plano, logo a comunicação e a empatia são essenciais nesse momento.

É gratificante encerrar o primeiro acolhimento com a gestante, quando essa sai do serviço de saúde com uma tranquilidade que não era percebida no primeiro momento. Mas para que isso ocorra é preciso que se estabeleça o vínculo desde o primeiro contato, não julgando, não deixando a gestante sem respostas, enfatizando muito sobre a possibilidade de não transmitir o vírus para o filho, pois esse é o maior medo vivenciado pelas gestantes. Para Hoeller et al, 2014, é necessário que ocorra uma interação entre profissionais de diferentes áreas e até mesmo entre diferentes níveis de assistência para que o acolhimento ocorra de uma 


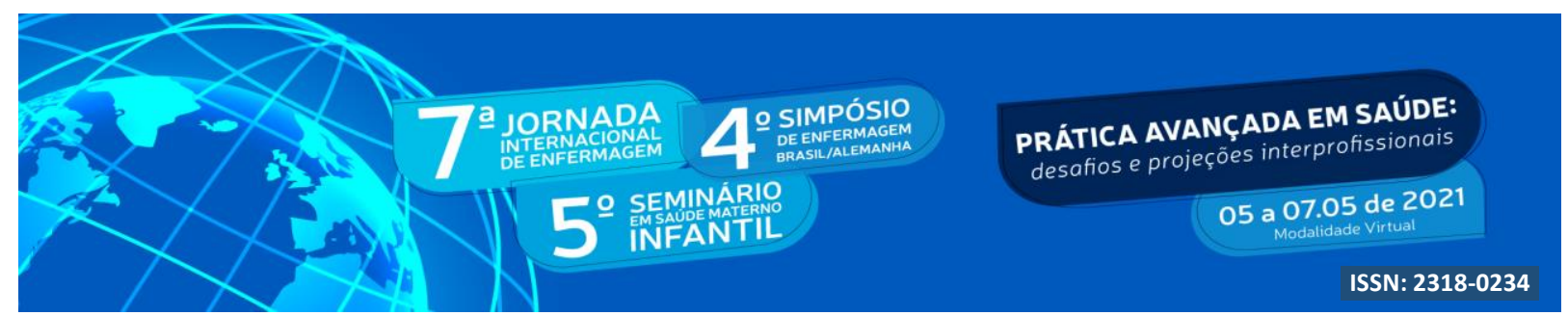

forma mais efetiva e integral.

\section{CONCLUSÃO}

A partir dessa experiência percebeu-se a importância de que os profissionai da saúde possam ser constantemente capacitados para o atendimento de gestantes com o diagnóstico de HIV/Aids, pois nesse primeiro contato já podemos estar contribuindo para uma boa adesão ao tratamento. Porém é necessário entender que não é só a clínica que se faz importante nesse momento de descoberta de diagnóstico, mas é fundamental nesse processo de acolher, ofertar um apoio emocional, pois a gestante precisa de um tempo para assimilar essa nova consição, de estar gerando uma nova vida, mas também de estar recebendo um diagnóstico para uma infecão ainda sem cura e que ainda causa muito medo, mas pricipalmente contribuir para que a gestante ou ainda pessoas do seu convívio possam desmitificar a infecção pelo HIV.

Podemos concluir que o profissional de saúde com uma visão ampliada, deve realizar um atendimento de forma humanizada, muitas vezes contribuindo para que se reestabeleçam as redes de apoio, se colocando à disposição de ouvir e acolher os familiares daquela gestante também. Acolher a gestante por inteiro, não a vendo apenas como uma mulher que está gerando uma criança ou apenas como alguém que possui uma doença, mas como uma pessoa que precisa ser cuidada e isso fortalecerá os vínculos com os profissionais da saúde, aumentando as chances dessa gestante dar seguimento no seu tratamento mesmo após a gestação. A vinculação do profissional com a gestante, otimiza o atendimento prestado e favorece para que os objetivos da equipe sejam alcançados.

\section{REFERÊNCIAS}

AIRES, R. da M. B., GUIMARÃES, L. B. E. Relato de Experiência de um Grupo de Residentes em Enfermagem Obstétrica Sobre Práticas de Organização do Processo de Trabalho. Rev enferm UFPE on line., Recife, fev., 2017.

ALVES, A.L.N. et al. Assistência de enfermagem à puérpera com síndrome da imunodeficiência humana adquirida. Braz. J. 


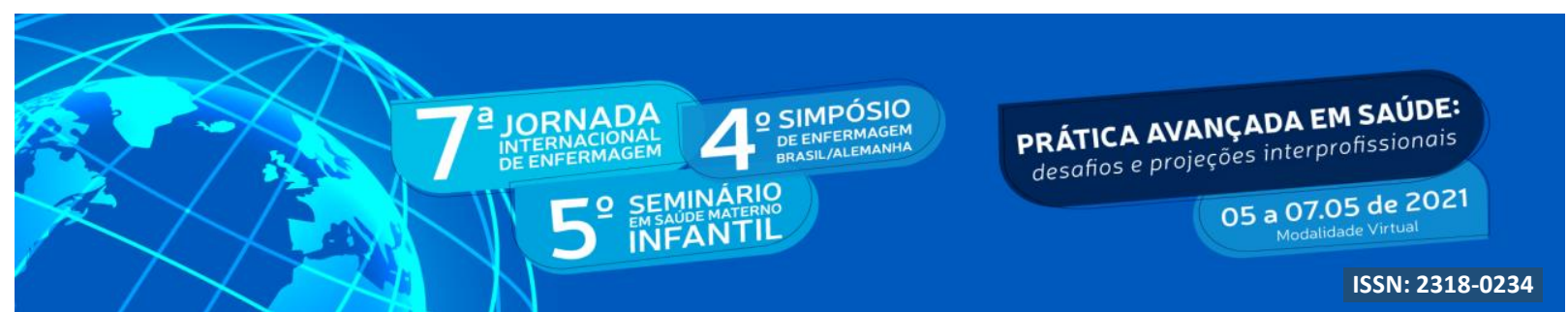

Hea. Rev., Curitiba, v. 3, n. 3, p.4023-4039 may./jun,2020. https://www.brazilianjournals.com/index.php/BJHR/article/view/9572/8055

AYALA, A.L.M.; MOREIRA, A.; FRANCELINO, G. Características Socioeconômicas e Fatores Associados à Positividade para o HIV em Gestantes de uma Cidade do Sul do Brasil. Revista APS [Internet]. 2016.

https://aps. ufjf.emnuvens.com.br/aps/article/view/2570/968

BARROS, V. L. et al. Fatores que interferem na adesão de gestantes com HIV/Aids à terapia antirretroviral. Revista Brasileira em Promoção da Saúde, vol. 24, núm. 4, octubrediciembre, 2011, pp. 396-403 Universidade de Fortaleza Fortaleza-Ceará, Brasil.

BRASIL. Ministério da saúde. Secretaria de Vigilância em Saúde. Boletim Epidemiológico: HIV/Aids. Bol. Epidemiol 2020

BRASIL. Ministério da Saúde. Secretaria de Atenção à Saúde, Departamento de Atenção Básica. Atenção ao pré-natal de baixo risco. Brasília: Ministério da Saúde, 2012.

BRASIL. Ministério da Saúde. Protocolos clínicos e medidas terapêuticas para prevenção da transmissão vertical de HIV Sífilis e Hepatites Virais, Brasília, DF, p. 25-37, 2019.

BRITO, A. M.; CASTILHO, E. A.; SZWARCWALD, C. L. AIDS e infecção pelo HIV no Brasil: uma epidemia multifacetada. Rev. Soc. Bras. Med. Trop., Uberaba, v. 34, n. 2, p. 207-217, Abri. 2001. <http://www.scielo.br/scielo.php?script=sci_arttext\&pid=S003786822001000200010\&lng=en\&nrm=iso>. Acesso em: 02 de março de 2021.

COSTA, M.M.R, et al. Qualidade de vida de indivíduos com HIV: revisão integrativa. Brazilian Journal of health Review. Braz. J. Hea. Rev., Curitiba, v. 2, n. 3, p. 1833-1842, mar./apr. 2019. ISSN 2595-6825. https://www.brazilianjournals.com/index.php/BJHR/article/view/1490

HOELLER, F. et al. Melhores práticas na atenção básica à saúde e os sentidos da integralidade.

Escola Anna Nery - Revista de Enfermagem, v. 17, n. 4, 2014. ISSN 1414-8145

SANTOS, E. I.; et. al. Evidências científicas brasileiras sobre adesão à terapia antirretroviral por pessoas que vivem com HIV/AIDS. Eletrônica Gestão \& Saúde. Rio de Janeiro. 2016. ISSN: 1982-478. http://publicacoes.unigranrio.edu.br/index.php/rcs/ Rev. article/view/2554. 


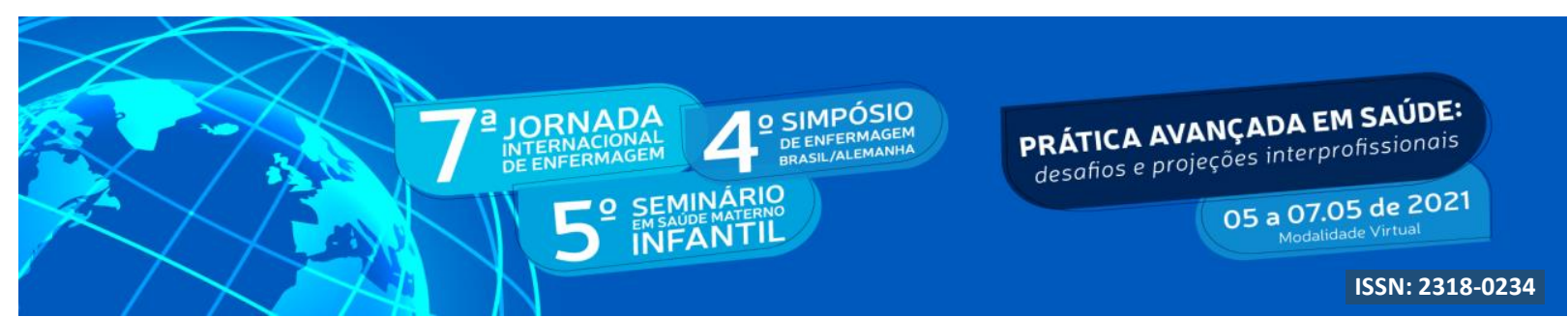

SECRETARIA DE MUNICÍPIO DE SAÚDE/RS. Relatório Anual de Gestão, 2019. http://www.santamaria.rs.gov.br/saude/658-relatorios-anuais-de-gestao

SILVA et al. Variáveis epidemiológica da infecção pelo o HIV em gestantes. Revista de Enfermagem da UFPI, v. 5, p. 34-39, jan./mar. 2016.

UNAIDS. Tratamento 2015. ONUSIDA, 2015. Disponível em: http://unaids.org.br/wp-content/uploads/2016/03/Tratamento-2015.pdf. Acesso em 15 de março de 2021. 